\title{
CGRP-monoclonal antibodies and SARS-CoV-2 vaccination
}

\author{
Francesca Schiano di Cola ${ }^{1}\left[\right.$ - Salvatore Caratozzolo ${ }^{1} \cdot$ Paolo Liberini $^{1} \cdot$ Alessandro Padovani $^{1} \cdot$ Renata Rao $^{1}$
}

Received: 8 October 2021 / Revised: 21 December 2021 / Accepted: 22 December 2021 / Published online: 8 January 2022

(c) The Author(s), under exclusive licence to Springer-Verlag GmbH Germany 2022

\section{Dear Sirs,}

The emergence and spread of the severe acute respiratory syndrome coronavirus 2 (SARS-CoV-2) has had a devastating impact globally. Since December 2019, control measures (personal protective equipment, physical distancing, contact tracing) have been implemeted to limit virus transmission. Due to its serious implications, the development of a vaccine against SARS-CoV-2 has been at the forefront of a worlwide initiative to stop the pandemic. Up to April 1st, three vaccines have been currently used in Italy: Pfizer/BioNTech Comirnaty, Moderna/mRNA-1273 and Astra-Zeneca/ Vaxzevria. These vaccines documented, respectively, a 94\%, $94.5 \%$ and $70.4 \%$ efficacy against the viral infection $[1,2]$. The most reported side effects were generally mild, ranging from injection site soreness, fatigue, headache, myalgia, chills, joint pain, fever, and nausea. These symptoms were also present in some of the participants during the clinical trials, and severe allergic reactions were reported in rare instances [1,2].

CGRP is involved in migraine pathophysiology, but also in the respiratory system and in immunological modulation [3]. Activated mast cells may release tryptase, which, in turn, activates the sensory neuron, stimulating CGRP release. Also bradykinin and prostaglandins, released by activated mast cells, may stimulate the release of CGRP from neurons. CGRP may also be produced by activated human B cells, in response to anti-IgM, IL-4 or nerve growth factor (NGF). In differentiated CD4+ T cells, CGRP induces elevation of cellular cAMP levels and inhibits the production of TNF $\alpha$ and IFN- $\gamma$ by TH 1 cells, but does not influence IL-4 production by TH 2 cells. In addition, CGRP may modulate adaptive immune responses by influencing the function of antigenpresenting cells.

Francesca Schiano di Cola

francescaschiano@hotmail.it

1 Neurology Unit, Department of Clinical and Experimental Sciences, University of Brescia, Piazzale Spedali Civili 1, 25123 Brescia, BS, Italy
Monoclonal antibodies targeting CGRP (CGRP-mAbs) have been found to be safe in clinical practice in terms of COVID-19 infection susceptibility and hospitalization/ symptoms [4]. No data are currently available regarding CGRP-mAbs and COVID-19 vaccines.

At the Headache Centre of the Department of Neurology at the University of Brescia/ASST Spedali Civili Brescia, 105 patients with migraine are currently on treatment with CGRP-mAbs, of whom 69 are receiving erenumab (29 patients on erenumab $70 \mathrm{mg} / 4$ weeks, 40 patients on erenumab $140 \mathrm{mg} / 4$ weeks), 28 on galcanezumab and 8 on fremanezumab. Out of 105 patients, 32 underwent COVID19 vaccinations while on treatment with CGRP-mAbs (8 patients on erenumab $70 \mathrm{mg} / 4$ weeks, 12 on erenumab 140 $\mathrm{mg} / 4$ weeks, 8 on galcanezumab, and 2 on fremanezumab). Eighteen patients received the Pfizer/BioNTech Comirnaty vaccine, 11 the Astra-Zeneca/Vaxzevria and 3 the Moderna/ mRNA-1273. All patients who received the Astra-Zeneca/ Vaxzevria vaccine only received the first dose, whereas all others received the full two vaccine administrations. A minimum of 10-14 days between CGRP-mAbs administration and COVID-19 vaccination was suggested. However, some patients did not comply to such recommendations. In particular, five patients waited, on average, less than 5 days between CGRP-mAbs administration and COVID-19 vaccination. None of our patients reported any serious adverse event regarding the vaccination, with most (18 patients) only reporting mild fever and fatigue. Six patients reported a temporary worsening of their usual headache, both in terms of intensity and frequency, lasting, on average, between 1 and 4 days. Side effects distribution was not affected by the type of CGRP-mAbs or vaccine, or by the time lapse between treatment administration and vaccination. CGRP-mAbs long-term efficacy was not affected. No patient reported COVID-19 infection following the vaccination.

Altogether, in our experience, no particular safety or efficacy issues concerning the interaction between CGRPmAbs and COVID-19 vaccines arose since the beginning of mass vaccination in January 2021. Given the cerebrovascular concerns that have accompanied both CGRP-mAbs 
and COVID-19 vaccines, together with the high volume of patients on treatment with CGRP-mAbs that will have to be vaccinated, we believe this preliminary report to be extremely helpful for neurologists and physicians in general.

Author contributions All authors contributed to the study conception and design. Material preparation, data collection and analysis were performed by FSC, SC and PL. The first draft of the manuscript was written by FSC and all authors commented on previous versions of the manuscript. All authors read and approved the final manuscript.

Funding The authors did not receive support from any organization for the submitted work.

Availability of data and materials The datasets generated during and/or analysed during the current study are available from the corresponding author on reasonable request.

\section{Declarations}

Conflicts of interest The authors have no financial or proprietary interests in any material discussed in this article.

Ethics approval Approval was obtained by the local ethics committee of the ASST Spedali Civili Hospital, Brescia (NP 3949, approved August 10, 2020).
Consent to participate Verbal informed consent was obtained prior to the interview.

Consent for publication N/A.

\section{References}

1. Anand P, Stahel VP (2021) The safety of COVID-19 mRNA vaccines: a review. Patient Saf Surg 15:20. https://doi-org.proxy. unibs.it/10.1186/s13037-021-00291-9

2. Loo KY, Letchumanan V, Ser HL et al (2021) COVID-19: insights into potential vaccines. Microorganisms 9(3):605. https://doi.org/ 10.3390/microorganisms9030605 (PMID: 33804162; PMCID: PMC8001762)

3. Holzmann B (2013) Modulation of immune responses by the neuropeptide CGRP. Amino Acids 45:1-7. https://doi-org.proxy. unibs.it/10.1007/s00726-011-1161-2

4. Caronna E, José Gallardo V, Alpuente A et al (2021) Safety of anti-CGRP monoclonal antibodies in patients with migraine during the COVID-19 pandemic: present and future implications. Neurologia. https://doi.org/10.1016/j.nrl.2021.03.003 (Epub ahead of print. PMID: 33832802; PMCID: PMC7973059) 